\title{
The Importance of Visual Identity Graphic Design in Cities
}

\author{
Zhang Chufan \\ XI'AN UNIVERSITY OF ARCHITECTURE AND ECHNOLOGY
}

\begin{abstract}
The identity system depends on tangible architecture, landscape roads and so on, but it doesn't equal to the supplement of its subject but an indispensable constituent part. As the finishing touch of the whole urban space, by means of design of elements such as symbols, graphs, characters and colors as well as integration of various factors, the identity system reflects the urban specialty, conveys urban features related to history, geography, culture and emotion. The identity system provides suitable space environment to people through application of concrete symbols, graphs, characters and colors, in addition, it also mirrors that the designing culture of a city equals to its design concept with the combination of the historical culture of this city. A modern city shall be provided with such function, that is, it shall possess a whole set of well-designed and scientific-planned indication mechanism to help those wandering pedestrians to indicate direction and find their way successfully. It shall not only serve the mass, realize inter-coordination among people, identity facility and space environment to make people's living environment more comfortable and convenient, but also it constitutes the vital carrier to spread local culture and model urban brand.
\end{abstract}

Key words- identity system; visual identity graph; historical culture

\section{INTRODUCTION}

The visual identity system is involved in the Public Information Symbols with expression of Sign design, Urban Identities and Sign Graphics commonly in English, and there is no a unified definition of it domestically. For a long time, people merely treat it as a kind of existence form for explanation of sign characters. Nowadays, people have gradually realized that it is more than a set of symbols but includes the existing environment, which is provided with unique regionalism, special individual functionality as well as representing form. It requires harmony with environment, and meanwhile should possess the function of distinct identification; it needs design with individualization without any conflict with the national Public Information Symbols System. The concept of "identity system" turned up nearly in the 1990s in China and people gradually realize its existing value. The none "identity" (Chinese characters "Dao" and "Shi") are composed of two verbs, namely, "lead" and "realize". The word "lead" means "guide", which acts as the action subject in the recognition system, while the word "realize" means "identity", which is treated as the passive target. The action object "realizes" and "identities" the passive target by means of "guiding" and "leading". However, it is more than a identification, but the design with systematization with integration of information on aspects of environment, architecture and graphs.

Starting from the Industrial Revolution, the industrialized development brings about fundamental changes on social structure, just like Chaplin's Modern Times, the large-scale production of machine imposes abrupt leap on personnel demand, and a good deal of labor demand impels large-scale migration rarely seen in the history of mankind, and also impels the change of the urban structure. In order to meet people's demand on urban functionality, comfortableness and convenience, urban construction enters into unprecedented development stage. 

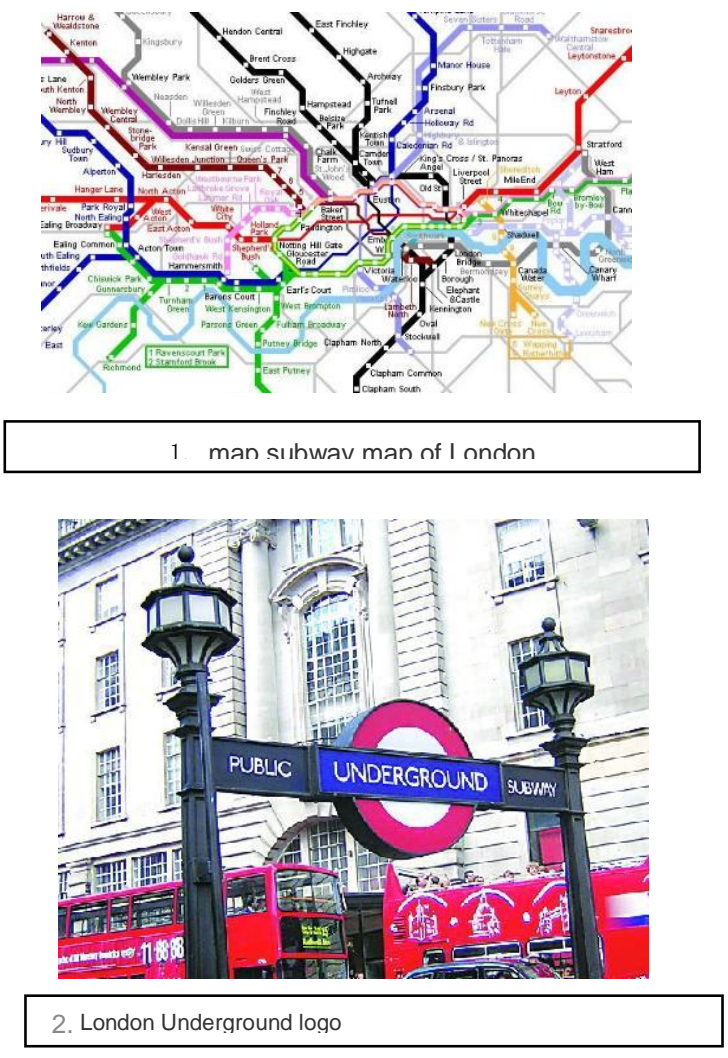

The design of visual identity system covers two design fields, namely, building landscape design and visual communication design, which is a comprehensive design research with coincidence of multi-disciplines. The design of visual identity system requires mastering knowledge of components related to materials and construction techniques with the scientificity and rationality of the identity system as more important aspect. "Le Corbusier published Tomorrow's City in 1992, and in this book, he planned a modern city with the population of three million people. In the city designed by him, a latticed road system is established, consisting of two super highways to form the Axis vertically and horizontally; the downtown area is composed by 24 skyscrapers with afforest and business service facilities around the architecture, and a complex communication network is formed by railway, highway and parking lot underground. Le Corbusier holds that the urban vitality is from exchanges on aspects of ideology, information, knowledge, intelligence as well as profession. Just think if there are no sufficient urban identities, problems will appear in its operation undoubtedly. Thanks to the enlargement of urban scale and further requirement on its functions, identification moves from obscure design field to the design foreground. City needs identification, and also needs the modern identification and recognition system that is harmonious with environment and can embellish the environment with scientific functions.

\section{History of Identity System 2.1 Origination}

"The movement of graphic design, starting from the 1920s, is the graphic reflection of the design of modernism. Differing from the movement of modernism architecture, this movement doesn't involve too much content related to social engineering and doesn't advocate design improves society and life of laboring people, but lays stress on the improvement of visual communication function on the graphic design. It creates the "world visual language" without necessity of characters, which makes use of design to reach the purpose of communication as the core of this movement. Of course, fundamentally, the movement of modern graphic design still shares the same core owed by the movement of modernism design with emphasis on the democratization of design as the purpose such as emphasis on utilization of graphs rather than the concept of character conveying for the ground of taking the demand of public with low cultural level, for this reason, there is difference between graphic design and other designs superficially, but all aspects of the modernism design movement share close connection with one another with similarity on thought as well as ideology. The movement of graphic design lasts over 20 years from its inception since 1920 s to 1940 s. Besides the font design, the test and attempt concentrates on realization on visual communication by means of graphs rather than characters, the constituent 
part of this movement is called 'Aesop system movement' ." Otto Neurath keeps a watchful eye on the pattern of visual communication and studies and design how to make it serve the society in a better way. He holds that the graphic design should provide means that the humankind can realize, and provide new patterns to promote the ideology and concept communication and exchange of mankind. He puts forward to utilize pictographic graphs to explain complex issues to the public, for example, the fertility rate issued by the nation can be represented by such graph with a baby sleeping in the cradle, while the death rate can be represented by black diamond with cross, which is unnecessary to merely adopt tedious figures to express it; the same to mentioned ones, the national military spending can also be represented by soldiers with guns. Graphs are vivid and can be understood by the public directly without too much explanation in words. Its exploration wins the support from the international modernism graphic design movement at that time. And thereafter, Neurath conducted joint exploration with other graphic design experts and set up the Transformation Team in Netherlands for the purpose of studying this method and accomplished this system. The new visual communication system with reminding as center was called "Vienna Method" in the beginning, and is called "Aesop System" finally.

They conduct optimization on those designed graphs, find out the best graphs in the communication function and ensure those graphs can be known and realized by great majority of people and are adopted at last. The achievement of the "Aesop System" and Transformation Team exists in the establishment of the world earliest graphic visual communication system with integrated system. This system consists of two parts, one is an independent graph equipped with international communicative function. A series of graphs through meticulous design and selection replace the frequently-used character conveying system in the past; the other refers to the graphic expressive system with combination of these graphs which is called "syntax" in English. This study result is of overriding significance for extensive use of graphs on aspects of public places, communication and transportation as well as telecommunications.

\subsection{Development \& Current Situation}

The ancients can get the accurate concept on time and space through observation on sun, moon and stars. And they also distinguish directions by means of observing the annual rings and leaves of trees as well as the Polaris. They gradually find out the laws of these natural phenomena and make them readable data, and afterwards, they invent compass, southward pointing cart as well as northward point acupuncture through long historical progress. After that, distinguishing direct doesn't merely depend on the nature.

There emerge cities evolving from city-states on the Mesopotamia since 3000 B.C, the analysis is conducted in view of people's cognitive aspect on urban environment, citizens' impression on the urban environment produces through several aspects. The first is urban pattern next to architecture, road and detailed features. Under condition of lack of urban identities, this type of sense of pattern becomes people's top selection. Some direction impression, shape impression, color impression and size impression produce in people's vision and the ancient seize environment in such way. After that, in the process of constant enlargement of cities, people begin to provide some objects of reference with special symbols or show their realization on environment through characters and graphs which can be known commonly. Some cultural symbols with special significance increase, and urban identity becomes one part of them. By the middle period in $20^{\text {th }}$ Century, urban booming perfects the identity, which becomes one vital means in the urban management.

There is increasing urban divisions and functional division with gradual refining, the 
traffic development changes the life and work types of the whole mankind. The old sailing might take several months even years, but you just spend several hours by air to realize the shift from one place to another. The traffic develops from original footing, horse and carriage to various kinds of vehicles such as buses and cars, airplanes as well as steamboats. Tremendous change fits the demand of modern people's life, and invention of vehicles and improvement of modes of transportation changes people's space-time concept together with the life style, which shortens distance among cities and brings about more rapid information communication, and people's activities and exchanges are more dependent on the graphic identity system existing in outer space. The traffic development constitutes the motive power to propel the constant improvement of graphic identity system of modern cities. In some traffic hub, there begins to emerge man-made graphs, which consist of symbols, image graphics as well as characters.

As far back as the middle period in $20^{\text {th }}$ Century, the western developed countries have realized the huge impact of the public environment identity system on the structure of urban development and construction, people start to apply the identity system into urban road planning, construction and reformation of roads and buildings together with public facilities. They make overall arrangements and unified planning on various factors existing in the urban development and construction with reasonable design and planning on aspects of matter identification on matters, mobility on people, medium on promotion and formal carrier on pattern so as to reach the harmonious development of integration of people, buildings, landscapes as well as cities.

Since the end of $20^{\text {th }}$ century, China's urban construction and reformation represents the peak state, urban portrait changes tremendously, which results in huge change in urban structure. Along with social progress and economic development, people begin to pay close attention to the environment on which they rely gradually. No matter the indoor and outdoor environment of buildings and landscapes or the urban planning, convenience, rationality and comfortableness are all demands required by people. Along with the constant increase of such demand, people begin to have increasing realization and comprehension on the graphic identity system, and as the extensive independent discipline of the visual conveying system, visual identity system achieves development by leaps and bounds in recent years. The overall development of China's economy falls behind that of western developed counties and so as to the development of graphic identity system, this is a chain relation, and the contradiction will show when the economic develops to certain degree. The economic development by leaps and bounds at this stage, the construction of infrastructure fails to meet people's actual demand far. The urban construction proceeds vigorously, but there are still many people lacking realization and comprehension on real significance of graphic identity system, which is to be realized and addressed.

Normative, reasonable and scientific design concept to make the graphic design under different circumstances to bear the contents in complex and huge urban environment. The graphics system design of visual identity is applied in the modern urban environment. As important central system in cities, the visual orientation, visual identity as well as image identity become the major standards to evaluate the overall image of the urban public environment.

\section{Consideration of Graphic System Design of Visual Identity}

3.1In view of psychology, the Gestalt Psychology is adopted here for illustration:

Gestalt Psychology-"perception" (visual perception) study, Gestalt is translated directly into "form", different from our implication, this is also called "form psychology". It originates from the study on aspect of visual perception, and the 
importance of Gestalt Psychology on the design art are expressed as follows:

“To begin with, it reveals human's perception with the visual perception in the highest flight in particular, which has no essential difference with more "advanced" thinking activities held by people usually, it doesn't require "relatively advanced" rational processing analysis, and the perception in itself possesses "thinking ability".

Second, in the process of the study of "thinking ability", many perception (mainly vision) laws are found, which are often applied in design with vial actual value. The main perception laws contain integrity, comprehensiveness, constancy as well as illusion.

Second, in the process of the study of "thinking ability", many perception (mainly vision) laws are found, which are often applied in design with vial actual value. The main perception laws contain integrity, comprehensiveness, constancy as well as illusion.

Finally, Gestalt psychologists also expand this theory into the study of creativity and creative thinking, they consider the artistic creation as a kind of process, and artists attempt to reach some kind of ideal image composition of graph under the tension with the pursuit of "favorable structure", as this pursuit draws near, this ideal image becomes more and more distinct, and the tension gets remission."

Along with changes of urban environment, people have different mental perception on environment, the visual identity system exists with the aim of correct guidance role. Through profound comprehension on the regionality, functionality and culture of environment, and with combination of deep research of peoples' mental perception on environment, the graphics system design can achieve significance on its existence. It is the real "people-oriented" design thinking that the design research is conducted from aspect of people's real feeling on urban space. The realization on details implied in the emotional space will make the urban design fill with intense emotion and make people's social life more rich and vivid full of vitality.
3.2 . In view of physiology, study the reflection of various sensory in human body on aspect of their capability to receive information, and distribute the design elements related to identity design in a more scientific way, this is conducive to the design of scientific and reasonable graphics system with visual identity: The gustatory nerves can receive $1 \%$ information;

The antennal nerves can receive $2 \%$ information;

The olfactory nerves can receive $4 \%$ information;

The hearing nerves can receive $8 \%$ information; The vision nerves can receive $83 \%$ information.

It is indicated from this data that vision takes up the overwhelming majority proportion on matters people get in touch with, which owns crucial influence. People receive main information through eyes, and this indicates that the original intention of this design is based on the extension with extroversion beyond the own functions of human body. Vision is the most dependent sensory function for the formative arts.

Understand people's physiological features clearly and utilize their own functional features, in this way can we design the graphics system with visual identity correctly and directly.

3.3. In view of design, it seems that the identity system belongs to the scope of graphic design, but as to the identity graphics system, it shall act on the space environment finally and it also shares close relation with specific space environment. As to people impacted by it, the system, firstly, should be provided with strong functionality, second, it involves many materials and technologies rather than graphic printed matters from fabrication methods to the implementation process, all this makes the identity graphics system differ from general graphic design. Now identity design has become one independent design category. It constitutes a more refining design category, reflects the design concept with "humanization" and reaches integration of people, architecture as 
well as landscape finally with respect to people-oriented design.

3.4. In view of public environment, the graphics system design with visual identity refers to the design activity against backdrop of public environment, which needs to express a series of image expressive functions explicitly on aspects of orientation, details, nature as well as principles. It mainly refers to the system design with visual graphics consisting of characters, graphs, symbols and forms. It doesn't exist in the environment in the isolated or dispersive form, but should be designed and studies through integrated and systematical design concept. Integrate its design style with the environment of the whole city to constitute the vital part of the urban image project. In view of form identity design, the study and exploration on relation among form, color and modeling is conducted on graphic and local scope, and the environment design focuses on exploration of the relation between architecture's functions and space form as well as structure in view of significance of architecture and places, and lays emphasis on environment space and landscape effect. In that way, in view of urban public environment, the identity design conducts the integrated design with visual imaging on many relevant elements, and realizes integration of various alienated aspects in the whole environment with the emphasis on the organization and management of the space order of public environment.

\section{Design Significance of Visual Identity Graphics Design}

The identity system in one city is just one tiny link in the whole city-planning construction, and people often fail to pay attention to it and ignore its existence and significance, of course, its existence cannot compete with those hypostatic building environment and landscapes obviously, but when we lose the identity system can we find it is difficult for us to move a single step just like the blind. The more advanced society develops, the bigger demand on identity system, and the more important it is. For example, we own a set of advanced drawing instrument but without instructions, some tools are never used and we have no any idea on them, no matter how advanced those tools are, they are merely decoration to us and we fail to give play of its advancement. The existence of identity graphics system acts as the instructions to help us to understand our city and guide us how to use this city.

The improvement of the identity graphics system is also the reflection of the urban civilized degree, which contains people's impression on overall environment of the city. Whether the visual environment information of this city is convenient and perfect or not imposes direct impact on urban development in future. No matter emerging cities or ancient ones, no matter modern architectures or ancient ones involving pavilions, terraces and pagodas, the visual identity graphics system becomes more and more important. The design and construction of the identity system is one discipline related to city management, which is the necessary link to reflect the urban image features. The identity system relies on hypostatic architectures, landscapes and roads, but it doesn't equal to the supplement of its subject but an indispensable constituent part.

The design of the visual identity graphics system is like a mirror to reflect the mental attitude of one city and one country in a specific stage. This mirrors the cultural quality, management level as well as civilization degree of this city from one side. As the finishing touch of the whole urban space, by means of design of elements such as symbols, graphs, characters and colors as well as integration of various factors, the identity system reflects the urban specialty, conveys urban features related to history, geography, culture and emotion.

\section{Conclusion}

Design is a kind of extension of urban culture, and the visual identity graphics design is a kind of new creation on historical culture, and also a preservation and re-realization on tradition from modern life. This makes people living in the modern cities to fully experience the glamour of traditional cultures, which shall the orientation of our efforts in our design work. 


\section{References}

[1] Zhou rui, Huang Yinjie,Zhou Yiliao. City Logo Design [M].Shanghai: Tongji University Press, 2004.

[2] Wang Shouzhi. World Graphic Design History [M].Beijing: China Youth Press, 2002.

[3] Liu Sha. Design Psychology of Art [M].Beijing: Tsinghua University Press, 2006.
[4] Bao Shidu,Wang Weiliang, Ge Rong. Identity-Oriented System Design Environment [M].Beijing: China Building Industry Press, 2007.

[5] Zhang Lili. Urban-Oriented Design[M].Shanghai: Shanghai University Press, 2005. 